\title{
MicroRNA-451 inhibits neuroblastoma proliferation, invasion and migration by targeting macrophage migration inhibitory factor
}

\author{
GENG LIU $^{1 *}$, ZHENGWEI XU ${ }^{2 *}$ and DINGJUN HAO ${ }^{2}$ \\ Departments of ${ }^{1}$ Hand Surgery and ${ }^{2}$ Spine Surgery, Hong-Hui Hospital, Xi'an Jiaotong University College of Medicine, \\ Xi'an, Shaanxi 710054, P.R. China
}

Received January 31, 2015; Accepted November 19, 2015

DOI: $10.3892 / \mathrm{mmr} .2016 .4770$

\begin{abstract}
Neuroblastoma (NB) is the most prevalent type of extracranial solid tumour in young children. To improve current understanding of the mechanisms, which modulate cancer cell proliferation, invasion and migration, investigations have focused on microRNAs (miRs), a class of small non-coding RNAs, which post-transcriptionally regulate gene expression during various crucial cell processes. The present study aimed to investigate the role of miR-451 in NB. Human NB tissue and adjacent normal tissue were surgically removed, and the expression of miR-451, and development and pathological characteristics of NB were investigated. The expression of miR-451 was reduced in the NB tissue, compared with that in the adjacent tissue, and correlations between the reduction in miR-451 and unfavourable variables included tumour size $(\mathrm{P}=0.0081)$, differentiation $(\mathrm{P}=0.0217)$, lymph node metastasis $(\mathrm{P}=0.0489)$, tumour-node-metastasis stage $(0.0220)$ and distant metastases $(\mathrm{P}=0.0201)$. Transfection of the SK-N-SH and GI-LA-N NB cell lines with miR-451 inhibited cell growth, invasion and migration. Furthermore, the present study demonstrated that macrophage migration inhibitory factor (MIF) was regulated directly by miR-451 and was a critical mediator of the biological effects of miR-451 in NB. The re-expression of MIF markedly reversed the carcinogenic inhibitory property of miR-451. These data provide a more detailed understanding of the essential role of miR-451 in NB, which relies on regulation of the expression of MIF.
\end{abstract}

Correspondence to: Professor Dingjun Hao, Department of Spine Surgery, Hong-Hui Hospital, Xi'an Jiaotong University College of Medicine, 76 Nanguo Road, Xi'an, Shaanxi 710054, P.R. China E-mail: dingjunhao196847@163.com

*Contributed equally

Key words: microRNA-451, neuroblastoma, macrophage migration inhibitory factor, tumourigenesis

\section{Introduction}

Neuroblastoma (NB), an embryonic malignancy derived from the neural crest, is one of the most common types of extracranial tumour in infants and children $(1,2)$. According to the Centers for Disease Control and Prevention, NB accounts for $\sim 7-10 \%$ of the cases of paediatric cancer and $15 \%$ of all paediatric cancer-associated mortality $(3,4)$. In addition, $\sim 60 \%$ of children with NB develop metastasis, with bone metastasis being particularly common following diagnosis (5). NB is characterized by unexpected clinical features, including spontaneous regression, maturation or aggressive progression (6). The 5-year survival rate for NB is $25-40 \%$ for patients $>1$ year of age with stage IV disease (7), and this poor prognosis is associated with the commonly advanced stage at diagnosis, which is due to the lack of effective screening programs and non-specific symptoms. Although multiple genetic and molecular lesions have been identified to be associated with NB tumourigenesis, the biological mechanisms governing the unusual behaviour of this tumour remain to be elucidated. Therefore, it is necessary to understand NB tumourigenesis and to identify novel therapeutic agents to improve the current management of NB.

MicroRNAs (miRs), a class of genes transcribing small, single-stranded, 19-24-nucleotide noncoding RNAs, have become the focus of substantial investigations. miRs are involved in a variety of biological processes, including cellular differentiation, apoptosis, metabolism and proliferation by silencing messenger RNAs (mRNAs) via binding to the 3'-untranslated region (3'UTR) of target genes $(8,9)$. Accumulating evidence from previous studies has demonstrated the erroneous expression patterns of miRs in several types of human cancer, and have demonstrated that they are involved in critical pathways linked to proliferation, cell cycle progression and metastasis (10-12). The human miR-451 gene is located within chromosome 17q11.2 (13). It has been found to be expressed in several human tissues and has a high level of expression in foetuses, compared with adults. Previous evidence has indicated that miR-451 can function as a tumour suppressor in nasopharyngeal carcinoma (14), hepatocellular carcinoma $(15,16)$ and bladder cancer $(17)$. Despite these previous findings concerning miR-451 and its important roles in carcinogenesis, there have been no reports to date regarding an association between miR-451 and NB. 
The aim of the present study was to evaluate the role of miR-451 in the development of NB. The results indicated that the aberrant expression of miR-451 is associated with NB tumourigenesis and linked with the biological behaviour of NB cells, indicating the potential use of miR-451 as a therapeutic target for NB.

\section{Materials and methods}

Patients and tissue samples. Human NB samples $(n=37$; male/female ratio, 17/20; age, $3 \pm 0.45$ years) and matched adjacent normal tissues were randomly obtained from patients who underwent curative resection at the Department of Spine Surgery, Hong-Hui Hospital (Xi'an, China) between 2012 and 2013. All samples were immediately frozen in liquid nitrogen. None of the patients were pretreated with radiotherapy or chemotherapy prior to surgery. The diagnoses of these tissue samples were all verified by pathologists at Hong-Hui hospital. All samples were obtained with informed consent, and the study protocol was approved by the Ethics Committee of Hong-Hui Hospital (Xi'an Jiaotong University College of Medicine, Xi'an, China).

RNA extraction and microRNA expression assay. All clinical specimens were stored at $-80^{\circ} \mathrm{C}$ and homogenized using a homogenizer (Invensys Systems APS, Albertslund, Denmark) at $500 \mathrm{x} \mathrm{g}$ at $4^{\circ} \mathrm{C}$ prior to extraction of RNA using a mirVa$\mathrm{na}^{\mathrm{TM}}$ miRNA Isolation kit (Applied Biosystems; Thermo Fisher Scientific, Inc., Waltham, MA, USA) by centrifugation $\left(12,000 \mathrm{x} \mathrm{g}, 4^{\circ} \mathrm{C}, 15 \mathrm{~min}\right)$, according to the manufacturer's protocol. The RNA concentration and quality were determined using a NanoDrop ND-1000 spectrophotometer (Thermo Fisher Scientific, Inc.), and $1 \mu \mathrm{g}$ RNA was used for reverse transcription (RT). A RT primer specific for miR-451 (5'-CGC TACGTAACGGCATGACAGTG(T)24-3'; Ambion, Austin, TX, USA) was used to produce cDNA from the miR in the RT procedure. Subsequently, quantitative-polymerase chain reaction (qPCR) was performed using SYBR Green PCR master mix (Applied Biosystems; Thermo Fisher Scientific, Inc.). PCR reaction mixture was composed of $1 \mu \mathrm{l} \mathrm{cDNA}, 0.5 \mu \mathrm{l}$ primer and $5 \mu \mathrm{l} \mathrm{SYBR}$ Green PCR master mix buffer (total volume, $10 \mu \mathrm{l})$. qPCR was performed over 30 cycles of $30 \mathrm{sec}$ at $98^{\circ} \mathrm{C}$, $90 \mathrm{sec}$ at $58^{\circ} \mathrm{C}$ and $20 \mathrm{sec}$ at $72^{\circ} \mathrm{C}$; with a final extension at $72^{\circ} \mathrm{C}$ for 5 min using a LightCycler ${ }^{\circledR}$ (Roche Diagnostics $\mathrm{GmbH}$, Mannheim, Germany). The primers were miR-451 forward, 5'-AAACCGTTACCATTACTGAGTT-3' and reverse, 5'-CGC TACGTAACGGCATGACAGTG-3'. The relative quantification of miR-451 was calculated using the $2^{-\Delta \Delta \mathrm{Cq}}$ method (18). The data were normalised using U6 as an internal control, and were measured relative to a calibrator sample as the external control.

Cell culture and transfection. The SK-N-SH and GI-LA-N human NB cell lines were obtained from the American Type Culture Collection and were grown in Eagle's minimum essential medium (Gibco; Thermo Fisher Scientific, Inc.), supplemented with $10 \%$ fetal bovine serum (FBS; Invitrogen; Thermo Fisher Scientific, Inc.) and $100 \mathrm{U} / \mathrm{ml}$ penicillin/streptomycin (Invitrogen), in a cell culture incubator at $37^{\circ} \mathrm{C}$ and $5 \% \mathrm{CO}_{2}$. The miR-451 mimics and control miR mimics were purchased from Shanghai GenePharma Co., Ltd. (Shanghai, China). The cells $\left(5 \times 10^{4}\right.$ cells/well) were transfected with the miR mimic $(30 \mathrm{nM})$ or the macrophage migration inhibitory factor (MIF) recombinant plasmid $(200 \mathrm{ng})$ using Lipofectamine ${ }^{\mathrm{TM}} 2000$ reagent (Invitrogen) at $37^{\circ} \mathrm{C}$, according to the manufacturer's protocol. All assays were performed $48 \mathrm{~h}$ following transfection.

Cell proliferation assay. To determine the cell proliferation capacity, the cells were monitored using cell growth curves. The cells were seeded into 96-well microplates $\left(10^{3}\right.$ cells/well), and cell viability was monitored using a 3-(4,5-dimethylthiazol-2-yl)-2,5-diphenyltetrazolium bromide (MTT) reduction assay (Sigma-Aldrich, St. Louis, MO, USA) at indicated time points (1, 2, 3, 4 and 5 days following seeding into plates). The absorbance value (A) was read at $570 \mathrm{~nm}$ using a Benchmark Microplate Reader (Bio-Rad Laboratories, Inc., Hercules, CA, USA).

Cell cycle analysis. The transfected cells $\left(5 \times 10^{4}\right.$ cells/well) were seeded into 6-well plates for $48 \mathrm{~h}$, collected by centrifugation (500 x g, $\left.4^{\circ} \mathrm{C}, 5 \mathrm{~min}\right)$, washed with cold phosphate-buffered saline (PBS) and fixed with $70 \%$ cold ethanol at $4^{\circ} \mathrm{C}$ overnight. The fixed cells were then collected, washed with PBS and resuspended in fluorescence-Activated Cell Sorting (FACS) solution, containing PBS, 0.1\% TritonX-100 (Sigma-Aldrich), $60 \mu \mathrm{g} / \mathrm{ml}$ propidium iodide (PI; Sigma-Aldrich), $0.1 \mathrm{mg} / \mathrm{ml}$ DNase-free RNase (Sigma-Aldrich) and $0.1 \%$ trisodium citrate (Sigma-Aldrich). After a final incubation on ice for $30 \mathrm{~min}$, cell cycle analysis was performed using a Becton Dickinson FACSCalibur flow cytometer (BD Biosciences, Franklin Lakes, NJ, USA) using CellQuest 5.2.1 (BD Biosciences) or ModFit 3.0 software (Verity Software House, Inc., Topsham, ME, USA) packages.

Apoptosis assay. An Annexin-V fluorescein isothiocyanate (FITC) Apoptosis kit (Cell Signaling Technology, Inc., Danvers, MA, USA) was used to evaluate apoptosis. Briefly, the cells were seeded into six-well culture plates at $5 \times 10^{4}$ cells/well and transfected for $48 \mathrm{~h}$, following which they were harvested by trypsinization and washed with cold PBS. Immediately following re-suspension of the cells in medium, Annexin-V FITC and PI buffers (KaiGi Technology, Guangzhou, China) were incubated with the cells for $30 \mathrm{~min}$ at $4^{\circ} \mathrm{C}$ in the dark. The Annexin V-FITC and PI signals were detected using flow cytometry (BD FACSCalibur; BD Biosciences).

Cell invasion assay. Transwell migration assays were performed using $6.5-\mathrm{mm}$ diameter cell culture inserts $(8-\mu \mathrm{m}$ pore size; BD Biosciences) in 24-well culture plates. A total of $2 \times 10^{4}$ suspended cells (per well) were seeded into the upper chamber of the Transwell insert, which was pre-coated with $1 \mathrm{mg} / \mathrm{ml}$ Matrigel (BD Biosciences). In the lower part of the chamber, $0.8 \mathrm{ml}$ medium with $20 \%$ FBS was added. The inserts were washed with PBS following incubation for 24 and $48 \mathrm{~h}$ at $37^{\circ} \mathrm{C}$, and the adherent cells on the membrane of the upper chamber were removed from the upper surface using a cotton swab. The cells on the lower chamber membrane were fixed in cold methanol and stained with $2 \%$ crystal violet (Sigma-Aldrich).

Scratch-wound assay. For the scratch-wound assay, the cells were seeded into 24 -well plates $\left(5 \times 10^{5}\right.$ per well) and cultured 
until confluent. The cell monolayers were wounded by manually scratching their surface with a $200-\mu 1$ pipette tip. The cells were then washed several times in culture medium to remove cell debris, and the culture was continued in fresh medium. After $12 \mathrm{~h}$ incubation at $37^{\circ} \mathrm{C}$, the migration of the cells was evaluated by counting the number of migrated cells under a phase contrast microscope (Eclipse 90i; Nikon, Tokyo, Japan).

Western blot analysis. The cells were lysed on ice using radioimmunoprecipitation assay buffer, containing $150 \mathrm{mM} \mathrm{NaCl}$, $1 \%$ sodium deoxycholate, $1 \%$ NP40, $0.1 \%$ sodium dodecyl sulfate, $50 \mathrm{mM}$ Tris ( $\mathrm{pH} 8.8$ ), $10 \mathrm{mM} \mathrm{NaF}, 1 \mathrm{X}$ protease inhibitors and phenylmethanesulfonyl fluoride. Protein concentrations were measured using a bicinchoninic acid assay method, and $30 \mu \mathrm{g}$ of protein was separated by $10 \%$ SDS-polyacrylamide gel electrophoresis (Sigma-Aldrich), followed by transfer onto polyvinylidene fluoride membranes (Durapore ${ }^{\circledR}$; Thermo Fisher Scientific) for analysis. The membranes were then blocked with $5 \%$ skimmed milk at room temperature for $1 \mathrm{~h}$ and washed with Tris-buffered saline containing Tween 20 three times for 5 min each. Rabbit anti-human MIF polyclonal antibody was used as the primary antibody (cat. no. ab7207; 1:500 dilution; Abcam, Cambridge, MA, USA). Rabbit anti-human GAPDH monoclonal antibody (cat. no. 5174; 1:1,000 dilution, Cell Signaling Technology, Inc.) was used as a control. The bands were detected using an Enhanced Chemiluminescence kit (GE Healthcare Life Sciences, Chalfont, UK) and visualised using a ChemiDoc XRS system (Bio-Rad Laboratories, Inc.).

Plasmid construction. To generate reporter constructs for the luciferase assays, the full-length 3'-untranslated region of MIF, as well as the mutant sequence of MIF, were synthesized using PCR. The sequences of the primers used were as follows: MIF 3'UTR, forward 5'-GGGGTACCCCAGAGCCGCAGG GACCCA-3' and reverse 5'-GAAGATCTTCAAGTCTCT AAACCGTTT-3'; and Mut MIF 3'UTR, forward 5'-TGG TGGGGAGAAATAAGTGCTTTAGAGACT-3' and reverse 5'-AGTCTCTAA AGCACTTATTTCTCCCCACCA-3'. The PCR-amplified MIF 3'UTR fragment was digested with $K p n I$ and $B g l \mathrm{II}$ restriction enzymes at $37^{\circ} \mathrm{C}$ for $6 \mathrm{~h}$ and then cloned into the restriction sites downstream of the luciferase open reading frame in the pGL3-promoter Luciferase vector (Invitrogen) at $4^{\circ} \mathrm{C}$ overnight using ligase.

The $3^{\prime}$-UTR deletion of MIF was amplified using PCR with the following primers: Forward 5'-GCCATCATGCCG ATGTTCATCG-3' and reverse 5'-CGGCTCTTAGGCGAA GGTGGAG-3'. The resulting PCR amplicons of MIF were cloned into the $\mathrm{T}$ vector (Promega Corporation, Madison, WI, USA). The clones were confirmed by sequencing.

Fluorescent reporter assays. For the Fluorescent reporter assay, the human NB cells were seeded into a 24-well plate $\left(1.5 \times 10^{5}\right.$ per well) and co-transfected with the miR mimics $(50 \mathrm{nM})$ and a reporter recombinant plasmid (100 ng) using Lipofectamine $^{\mathrm{TM}} 2000$ reagent (Invitrogen; Thermo Fisher Scientific, Inc.), according to the manufacturer's protocol. Luciferase activity was analysed $48 \mathrm{~h}$ following co-transfection using dual luciferase assays (Promega Corp.), and values were normalised against the activity of the Renilla luciferase gene.
Statistical analysis. All values are expressed as the mean \pm standard deviation from at least three repeated individual experiments for each group. The data obtained in the present study were analyzed using the statistical package SPSS version 17.0 (SPSS Inc., Chicago, IL, USA). Significant differences were analysed using Student's $t$-test or $\chi^{2}$ analysis for comparisons between two groups, and one-way analyses of variance or the non-parametric Kruskal-Wallis $\mathrm{H}$ test for multiple comparisons. $\mathrm{P}<0.05$ was considered to indicate a statistically significant difference.

\section{Results}

Expression of miR-451 in human NB tissues. To investigate the pathogenicity of miR-451 in the development and progression of NB, 37 NB tissue samples were analysed using RT-qPCR. The clinicopathologic data for 37 patients are presented in Table I. Based on the overall expression level of miR-451, the NB specimens were divided into two groups, a high miR-451 expression level group and a low expression level group. The results showed that the expression of miR-451 in the NB tissues was significantly lower, compared with that in the matched paraneoplastic tissues $(\mathrm{P}=0.0001)$. Additionally, significant correlations were found between the reduction in miR-451 and unfavourable variables, including carcinoma size $(\mathrm{P}=0.0081)$, level of differentiation $(\mathrm{P}=0.0217)$, TNM stage $(\mathrm{P}=0.0220)$, lymph node metastasis $(\mathrm{P}=0.0489)$ and distant metastases $(\mathrm{P}=0.0201)$. No significant difference was observed when the groups were divided by the clinicopathologic features of gender and age.

miR-451 retards cell growth and induces $G 1$ arrest in human $N B$ cells. To investigate the role of miR-451 in human NB, miR-451 was overexpressed in SK-N-SH and GI-LA-N human NB cells by transfection with miR-451 mimics. The RT-qPCR results showed that transfection with the miR-451 mimics markedly elevated the levels of miR-451 in the SK-N-SH and GI-LA-N cells (Fig. 1A). The MTT assays showed that the overexpression of miR-451 significantly inhibited the growth of the SK-N-SH and GI-LA-N cells, and this effect increased with time (Fig. 1B). To further elucidate the antigrowth mechanism of miR-451 in NB cells, the present study analysed cell cycle and apoptosis. Overexpression of miR-451 in the NB cells significantly increased the percentage of cells in the $G_{0} / G_{1}$ phase of the cell cycle, compared with the control group (Fig. 1C). However, no significant difference were observed in the rates of apoptosis following transfection with the miR-451 mimics in the SK-N-SH and GI-LA-N cells (Fig. 1D).

miR-451 inhibits the invasion and migration of $N B$ cells in vitro. The effect of miR-451 on the invasion and migration of human NB cells was evaluated using Transwell and scratch-wound assays. The Transwell assays showed that transfection with the miR-451 mimics reduced the number of invasive cells in the SK-N-SH and GI-LA-N cell lines, compared with the control group (Fig. 2A and B). In addition, the scratch-wound assays also revealed that the migratory abilities of the SK-N-SH and GI-LA-N cells were significantly reduced following transfection with the miR-451 mimics (Fig. 2C and D). 
Table I. Correlations between the expression of miR-451 in neuroblastoma and clinical characteristics.

\begin{tabular}{|c|c|c|c|c|}
\hline \multirow[b]{2}{*}{ Characteristic } & \multirow[b]{2}{*}{$\mathrm{n}$} & \multicolumn{2}{|c|}{$\begin{array}{l}\text { Relative expression of } \\
\text { miR-451 }\end{array}$} & \multirow[b]{2}{*}{ P-value } \\
\hline & & Low & High & \\
\hline Paraneoplastic tissues & 37 & 12 & 25 & 0.0001 \\
\hline Carcinoma tissue & 37 & 30 & 7 & \\
\hline Gender & & & & 0.5148 \\
\hline Male & 17 & 7 & 10 & \\
\hline Female & 20 & 11 & 9 & \\
\hline Age (years) & & & & 1.0000 \\
\hline$>5$ & 13 & 6 & 7 & \\
\hline$\leqq 5$ & 24 & 10 & 14 & \\
\hline Size of carcinoma & & & & 0.0081 \\
\hline$>3 \mathrm{~cm}$ & 18 & 14 & 4 & \\
\hline$\leqq 3 \mathrm{~cm}$ & 19 & 6 & 13 & \\
\hline Degree of differentiation & & & & 0.0217 \\
\hline Well and moderately differentiated & 19 & 5 & 14 & \\
\hline Poorly differentiated & 18 & 12 & 6 & \\
\hline TNM stage & & & & 0.0220 \\
\hline I-II & 20 & 5 & 15 & \\
\hline III-IV & 17 & 11 & 6 & \\
\hline Lymph node metastasis & & & & 0.0489 \\
\hline Negative & 21 & 7 & 14 & \\
\hline Positive & 16 & 11 & 5 & \\
\hline Distant metastasis & & & & 0.0201 \\
\hline Negative & 16 & 4 & 12 & \\
\hline Positive & 21 & 14 & 7 & \\
\hline
\end{tabular}

Differences between variables were assessed using Fisher's exact test. miR, microRNA; TNM, tumor-node-metastasis.

A

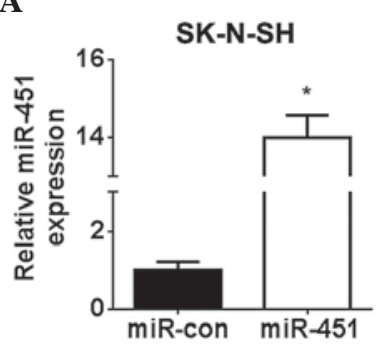

C

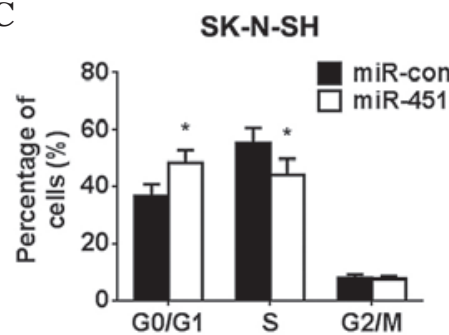

GI-LA-N

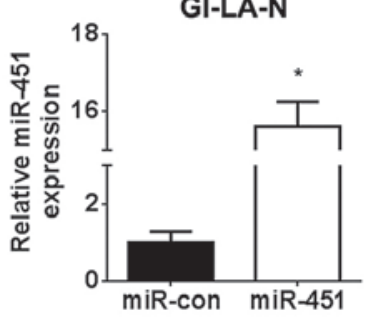

GI-LA-N

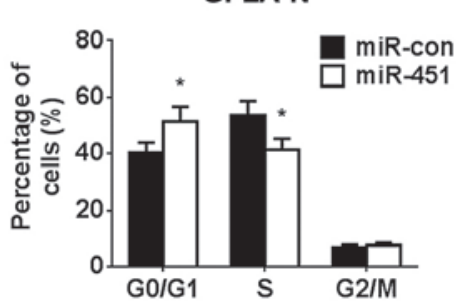

B
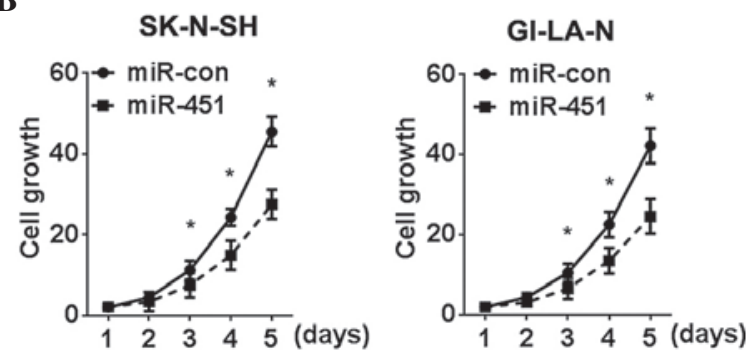

D

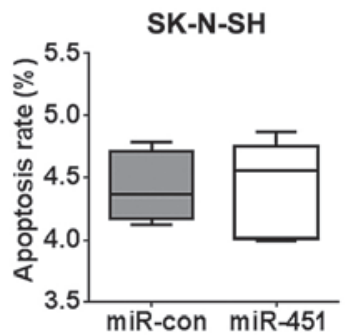

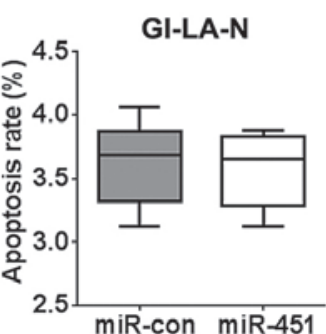

Figure 1. Effect of miR-451 on SK-N-SH and GI-LA-N cell proliferation. The SK-N-SH and GI-LA-N cells were transfected with control miR mimics or miR-451 for $48 \mathrm{~h}$. (A) Mature miR-451 expression was measured using reverse transcription-quantitative polymerase chain reaction analysis. U6 was used as an internal reference. (B) Cell growth curves for SK-N-SH and GI-LA-N cells were determined using a 3-(4,5-dimethylthiazol-2-yl)-2,5-diphenyltetrazolium bromide assay. Absorbance on day 1 was assigned a value of 1. (C and D) Cell cycle and apoptosis were analysed using flow cytometry. Data are expressed as the mean \pm standard deviation $(\mathrm{n}=5)$. ${ }^{*} \mathrm{P}<0.05$, vs. control group. miR, microRNA; con, control. 
A

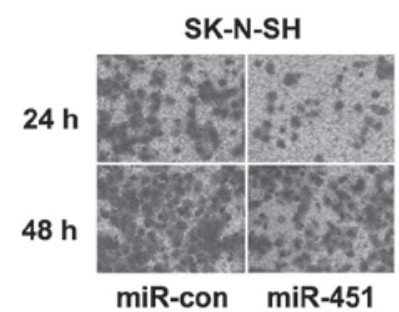

GI-LA-N

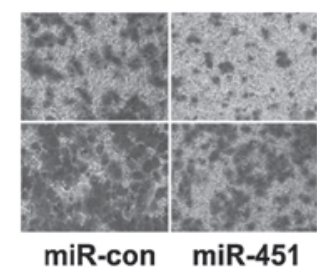

B

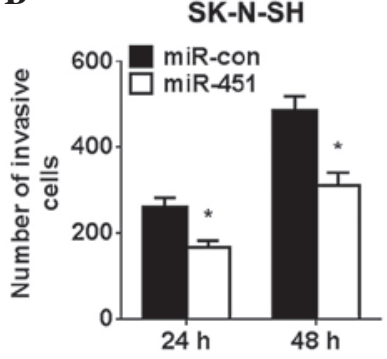

D

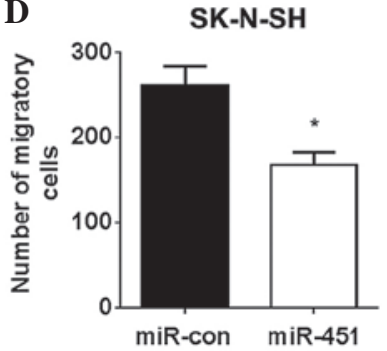

GI-LA-N
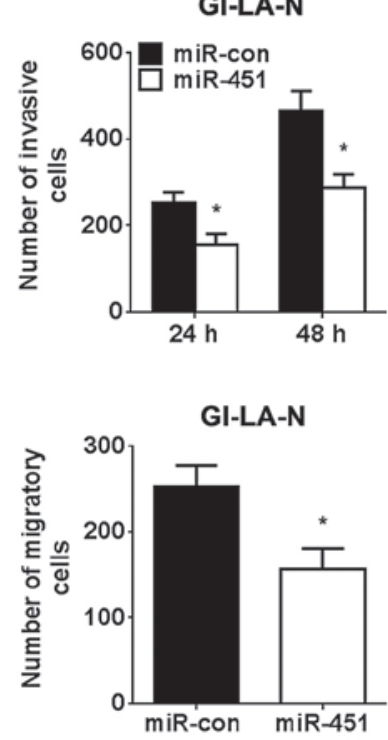

Figure 2. Effects of miR-451 on the migration and invasion ability of NB cells. The SK-N-SH and GI-LA-N cells were transfected with control miRNA mimics or miR-451 for $48 \mathrm{~h}$. (A) Representative images of the Transwell migration assay following incubation for 24 and $48 \mathrm{~h}$ (magnification, $\mathrm{x} 200$ ). (B) Quantification of the number of transmembrane cells, by counting in five high-power fields in each chamber. (C) Representative images were captured at 0 and 12 h to assess the cell migration into the wound space (magnification, x100). (D) Quantification of the number of migrated cells by counting in five high-power fields. Data are expressed as the mean \pm standard deviation $(n=5)$. ${ }^{*} \mathrm{P}<0.05$, vs. control group. miR, microRNA; con, control.

A

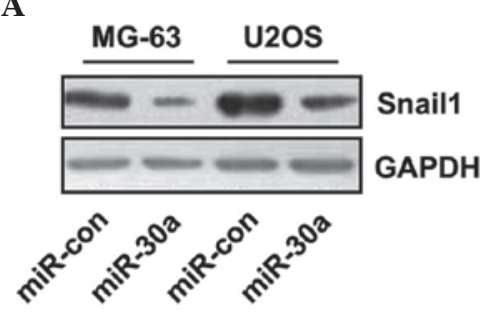

B

Snail1 3'UTR
miR-30a
Mut Snail1 3'UTR
miR-30a

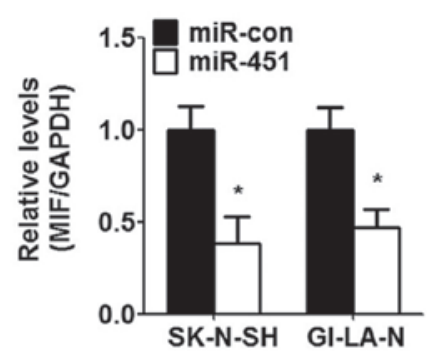

C

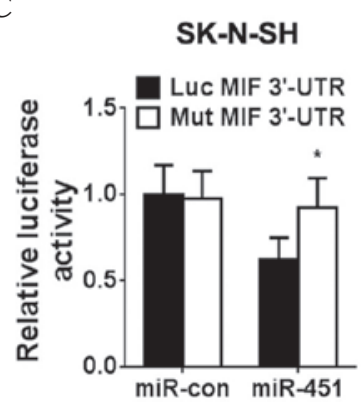

D

GI-LA-N

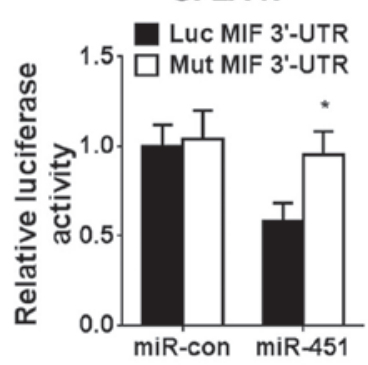

Figure 3. miR-451 directly targets MIF and inhibits its expression. The SK-N-SH and GI-LA-N cells were transfected with control miRNA mimics or miR-451 for $48 \mathrm{~h}$. (A) Western blot analysis was used to detect the protein expression of MIF. GAPDH was used as an internal control. Relative expression of MIF was calculated based on densitometric analysis of band intensities. (B) Full-length 3'-UTR sequences of wild-type MIF and mutant MIF were cloned into the pGL3-promoter luciferase vector. The predicted base pairing between the miR-451 and the 3'-UTR sequence of MIF is shown (solid lines indicate matching base pairs; crosses represent non-matching base pairs). (C and D) SK-N-SH and GI-LA-N cells were co-transfected with the luciferase reporter plasmid carrying the full-length 3'-UTR sequences of wild-type MIF or mutant MIF and the miR-451 mimics. The data are expressed as the mean \pm standard deviation $(\mathrm{n}=5)$. ${ }^{*} \mathrm{P}<0.05$, vs. group comprising the luciferase reporter plasmid carrying full-length 3 '-UTR sequences of wild-type MIF. miR, microRNA; con, control; MIF, macrophage migration inhibitory factor; 3'-UTR, 3'-untranslated region.

MIF is a direct target of miR-451 in human NB cells. As miRs exert their function predominantly through the inhibition of target genes, the present study detected the target of miR-451 in NB cells. Previous studies have reported that MIF serves as a target of miR-451 post-transcriptional repression in gastrointestinal and nasopharyngeal carcinoma $(14,19)$. To demonstrate whether miR-451 regulated the expression of MIF in NB, the SK-N-SH and GI-LA-N cells were transfected with miR-451 mimics. Western blot analysis confirmed that transfection with the miR-451 mimics significantly reduced the protein expression of MIF in the NB cells (Fig. 3A). To demonstrate that the inverse correlation between miR-451 and the expression of MIF 
A

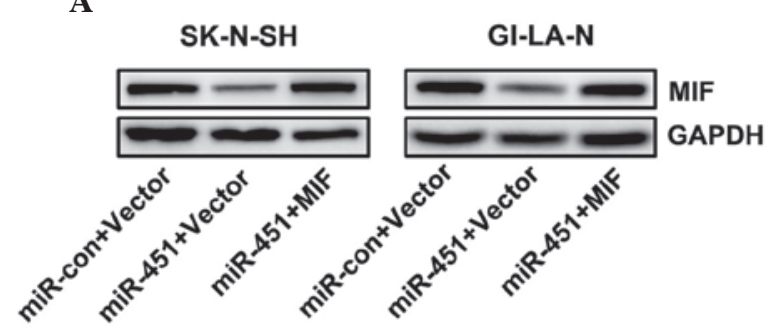

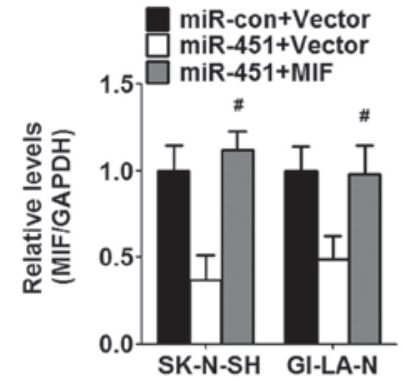

C

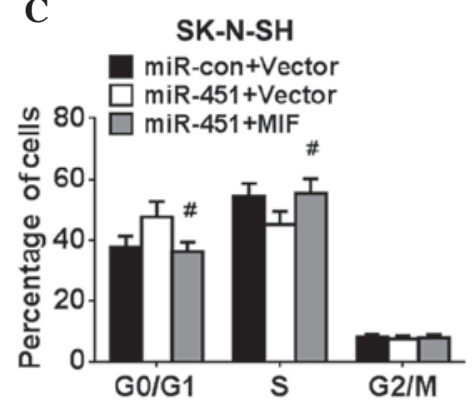

$\mathbf{E}$

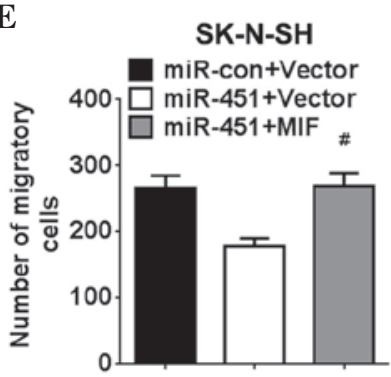

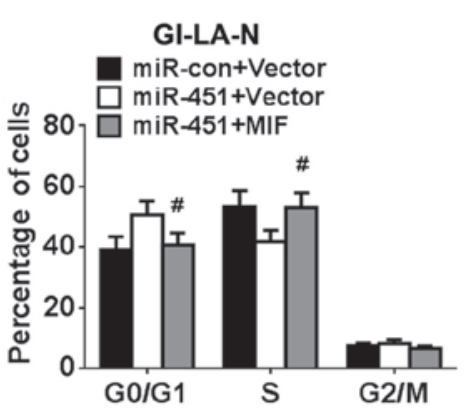

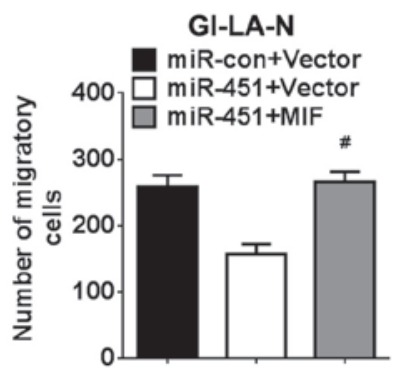

Figure 4. Ectopic expression of MIF can reverse the inhibitory tumourigenic properties of miR-451. The SK-N-SH and GI-LA-N cells were co-transfected with control miRNA mimics or miR-451 mimics and the empty vector or MIF recombinant plasmid for $48 \mathrm{~h}$. (A) Western blot analysis was used to detect the protein expression of MIF. GAPDH was used as an internal control. Relative expression of MIF was calculated based on densitometric analysis of band intensities. (B) Cell growth curves for NB cells were determined using a 3-(4,5-dimethylthiazol-2-yl)-2,5-diphenyltetrazolium bromide assay. (C) Cell cycle progression was analysed using flow cytometry. (D) Transwell assays were performed to determine the number of invasive cells. (E) Scratch-wound assays were used to determine the number of migrated cells. Data are expressed as the mean \pm standard deviation $(\mathrm{n}=5)$. ${ }^{\prime} \mathrm{P}<0.05$, vs. miR-451+Vector group. miR, microRNA; con, control; MIF, macrophage migration inhibitory factor.

in NB cells was due to a direct interaction, the potential seed sequence for miR-451 in the 3'UTR region of MIF was analysed, and the wild-type and mutant MIF 3'UTR fragments were cloned into a luciferase reporter gene system (Fig. 3B). The MIF 3'-UTR luciferase reporter plasmid and the miR-451 or control mimics were co-transfected into the SK-N-SH and GI-LA-N cells. The results revealed a 38.5 and $46.9 \%$ reduction in luciferase activity in the miR-451-transfected SK-N-SH cells and GI-LA-N cells, respectively, compared with the cells transfected with the control, respectively. However, no alteration in luciferase activity was detected with the mutant MIF 3'-UTR luciferase reporter plasmid (Fig. 3C and D).

Ectopic expression of MIF reverses the inhibitory property of $m i R-451$. To examine whether the repression of miR-451 on NB cell growth and invasion was mediated through MIF, the present study co-transfected the NB cells with expression vectors for MIF lacking the respective 3'UTR, or empty vectors, with the miR-451 mimics. Western blot analysis demonstrated increased protein expression of MIF in the NB cells co-transfected with this MIF vector, compared with those transfected with the empty vector (Fig. 4A). The re-expression of MIF led to the apparent rescue of NB cell proliferation, determined from the results of the MTT assay, with no significant differences, compared with the control (Fig. 4B). Cell cycle analysis revealed that the re-expression of MIF decreased the percentage of cells in the $G_{0} / G_{1}$ phase and reversed the growth inhibitory effect of miR-451 (Fig. 4C). Similarly, the Transwell and scratch-wound assays showed that the re-expression of MIF reversed the inhibitory effects of miR-451 on invasion and migration (Fig. 4D and E).

\section{Discussion}

NB carcinogenesis is a complex multistep process, which involves gene mutation, epithelial-mesenchymal transition, immune escape and tumour microenvironmental stimuli. It is characterized by clinical features, including spontaneous regression, maturation or aggressive progression (20). Over the past few years, various miR profiles have indicated that alterations in the expression levels of particular miRs may be critical in cancer initiation and progression, including the progression of tumourigenesis in NB (21). 
The dysregulated expression of miRs has been found to correlate with NB growth, invasion and migration, thus reinforcing the importance of miR biology in NB-associated tumourigenesis $(22,23)$.

In the present study, $\mathrm{t}$ miR-451 was found to be downregulated in NB tissues, and tumours of patients with lower levels of miR-451 tended to be larger, poorly differentiated and associated with metastasis. These data suggested a potential anti-oncogenic function for miR-451. The overexpression of miR-451 in NB cells decreased cell proliferation, invasion and migration, and there was a marked upregulation in the number of cells in the $G_{0} / G_{1}$ phase. These results were consistent with previous studies demonstrating that miR-451 acts as a tumour suppressor gene in carcinogenesis $(13,24)$. Therefore, the expression of miR-451 may be an important indicator for NB carcinogenesis and growth. The data obtained in the present study indicated a novel regulation function of miR-451 in NB cells, and suggested that miR-451 may be important in the pathogenesis of NB.

Furthermore, the present study confirmed MIF as a direct target gene of miR-451, and showed that miR-451 negatively regulated the expression of MIF expression by directly targeting the 3'UTR of MIF mRNA in the NB cells. MIF is considered to be a multifunctional cytokine, secreted by a variety of cells, including macrophages, lymphocytes, eosinophils, epithelial cells and endothelial cells (25). It is predominantly immunoregulatory, serving important roles in inflammation, and in cell-mediated and innate immunity (26-28). Consequently, MIF exerts a dominant effect in diseases that are characterized by pro-inflammatory pathways. MIF has now been recognized as a protumourigenic factor, and its overexpression has been found in human melanoma (29), breast carcinoma (30), pancreatic cancer (31), hepatocellular carcinoma (32), epithelial ovarian cancer (33), nasopharyngeal carcinoma (34), and gastric and colon cancer (35). A previous study provided direct evidence that MIF is associated with human NB growth and metastasis, and also demonstrated that MIF function is mediated by inducing the expression of N-Myc via activation of the extracellular signal-regulated kinase signalling pathway and increased expression levels of interleukin-8 and vascular endothelial growth factor (36). These data suggest that the overexpression of MIF is involved in NB carcinogenesis $(36,37)$. In addition, the present demonstrated that the inhibitory effect of miR-451 on the proliferation, invasion and migration of NB cells was reversed by overexpressing MIF, suggesting that miR-451 inhibited NB tumourigenesis by suppressing the expression of MIF.

The elucidation of the modulatory mechanism of specific miRs in cancer cell lines is essential to determining the prognostic and therapeutic values assigned to the miR in the tumour. The present study is the first, to the best of our knowledge, to show the clinical characteristics of the expression of miR-451 expression in NB. The present study also identified the important molecular mechanism by which miR-451 exerts its negative effects on proliferation, invasion and migration in human NB cells, via the concomitant suppression of MIF. This novel miR-451/MIF pathway provides further insight into the molecular mechanisms, which regulate the progression of $\mathrm{NB}$ and indicates novel therapeutic targets for the treatment of patients with NB.

\section{References}

1. Cheung NK and Dyer MA: Neuroblastoma: Developmental biology, cancer genomics and immunotherapy. Nat Rev Cancer 13: 397-411, 2013.

2. Li Y, Li W, Zhang JG, Li HY and Li YM: Downregulation of tumor suppressor menin by miR-421 promotes proliferation and migration of neuroblastoma. Tumour Biol 35: 10011-10017, 2014.

3. Maris JM, Hogarty MD, Bagatell R and Cohn SL: Neuroblastoma. Lancet 369: 2106-2120, 2007.

4. Li D, Mei H, Qi M, Yang D, Zhao X, Xiang X, Pu J, Huang K, Zheng $\mathrm{L}$ and Tong Q: FOXD3 is a novel tumor suppressor that affects growth, invasion, metastasis and angiogenesis of neuroblastoma. Oncotarget 4: 2021-2044, 2013.

5. Qiao J, Lee S, Paul P, Qiao L, Taylor CJ, Schlegel C, Colon NC and Chung DH: Akt2 regulates metastatic potential in neuroblastoma. PLoS One 8: e56382, 2013.

6. Kim YS, Lee HA, Lim JY, Kim Y, Jung CH, Yoo SH and Kim Y: $\beta$-Carotene inhibits neuroblastoma cell invasion and metastasis in vitro and in vivo by decreasing level of hypoxia-inducible factor-1 $\alpha$. J Nutr Biochem 25: 655-664, 2014.

7. Chen X, Pan M, Han L, Lu H, Hao X and Dong Q: MiR-338-3p suppresses neuroblastoma proliferation, invasion and migration through targeting PREX2a. FEBS Lett 587: 3729-3737, 2013.

8. Farh KK, Grimson A, Jan C, Lewis BP, Johnston WK, Lim LP, Burge CB and Bartel DP: The widespread impact of mammalian MicroRNAs on mRNA repression and evolution. Science 310: 1817-1821, 2005.

9. Wu WK, Lee CW, Cho CH, Fan D, Wu K, Yu J and Sung JJ: MicroRNA dysregulation in gastric cancer: A new player enters the game. Oncogene 29: 5761-5771, 2010.

10. Sassen S, Miska EA and Caldas C: MicroRNA: Implications for cancer. Virchows Arch 452: 1-10, 2008.

11. Bartel DP: MicroRNAs: Genomics, biogenesis, mechanism and function. Cell 116: 281-297, 2004.

12. Ambros V: The functions of animal microRNAs. Nature 431: 350-355, 2004.

13. Liu D, Liu C, Wang X, Ingvarsson S and Chen H: MicroRNA-451 suppresses tumor cell growth by down-regulating IL6R gene expression. Cancer Epidemiol 38: 85-92, 2014.

14. Liu N, Jiang N, Guo R, Jiang W, He QM, Xu YF, Li YQ, Tang LL, Mao YP, Sun Y and Ma J: MiR-451 inhibits cell growth and invasion by targeting MIF and is associated with survival in nasopharyngeal carcinoma. Mol Cancer 12: 123, 2013.

15. Liu X, Zhang X, Xiang J, Lv Y and Shi J: miR-451: Potential role as tumor suppressor of human hepatoma cell growth and invasion. Int J Oncol 45: 739-745, 2014.

16. Lv G, Hu Z, Tie Y, Du J, Fu H, Gao X and Zheng X: microRNA-451 regulates activating transcription factor 2 expression and inhibits liver cancer cell migration. Oncol Rep 32: 1021-1028, 2014.

17. Zeng T, Peng L, Chao C, Fu B, Wang G, Wang Y and Zhu X: MiR-451 inhibits invasion and proliferation of bladder cancer by regulating EMT. Int J Clin Exp Pathol 7: 7653-7662, 2014.

18. Wang Y, Liang Z, Gao Y, et al: Factors influencing circulating MicroRNA level in the studies of hepatocellular carcinoma biomarker. Neoplasma 62: 798-804, 2015.

19. Bandres E, Bitarte N, Arias F, Agorreta J, Fortes P, Agirre X, Zarate R, Diaz-Gonzalez JA, Ramirez N, Sola JJ, et al: microRNA-451 regulates macrophage migration inhibitory factor production and proliferation of gastrointestinal cancer cells. Clin Cancer Res 15: 2281-2290, 2009.

20. Guo J, Dong Q, Fang Z, et al: Identification of miRNAs that are associated with tumor metastasis in neuroblastoma. Cancer Biol Ther 9: 446-452, 2010.

21. Xin C, Buhe B, Hongting L, Chuanmin Y, Xiwei H, Hong Z, Lulu H, Qian D and Renjie W: MicroRNA-15a promotes neuroblastoma migration by targeting reversion-inducing cysteine-rich protein with Kazal motifs (RECK) and regulating matrix metalloproteinase-9 expression. FEBS J 280: 855-866, 2013.

22. Zhao D, Tian Y, Li P, et al: MicroRNA-203 inhibits the malignant progression of neuroblastoma by targeting Sam68. Mol Med Rep 12: 5554-5560, 2015.

23. Wu K, Yang L, Chen J, et al: miR-362-5p inhibits proliferation and migration of neuroblastoma cells by targeting phosphatidylinositol 3-kinase-C2 $\beta$. FEBS Lett 589: 1911-1919, 2015.

24. Su Z, Zhao J, Rong Z, Geng W and Wang Z: MiR-451, a potential prognostic biomarker and tumor suppressor for gastric cancer. Int J Clin Exp Pathol 8: 9154-9160, 2015. 
25. Guo Y, Hou J, Luo Y and Wang D: Functional disruption of macrophage migration inhibitory factor (MIF) suppresses proliferation of human H460 lung cancer cells by caspase-dependent apoptosis. Cancer Cell Int 13: 28, 2013.

26. Conroy H, Mawhinney L and Donnelly SC: Inflammation and cancer: Macrophage migration inhibitory factor (MIF) - the potential missing link. QJM 103: 831-836, 2010.

27. Cooke G, Armstrong ME and Donnelly SC: Macrophage migration inhibitory factor (MIF), enzymatic activity and the inflammatory response. Biofactors 35: 165-168, 2009.

28. Bucala R and Donnelly SC: Macrophage migration inhibitory factor: A probable link between inflammation and cancer. Immunity 26: 281-285, 2007.

29. Oliveira CS, de Bock CE, Molloy TJ, Sadeqzadeh E, Geng XY, Hersey P, Zhang XD and Thorne RF: Macrophage migration inhibitory factor engages PI3K/Akt signalling and is a prognostic factor in metastatic melanoma. BMC Cancer 14: 630, 2014.

30. Richard V, Kindt N, Decaestecker C, Gabius HJ, Laurent G, Noël JC and Saussez S: Involvement of macrophage migration inhibitory factor and its receptor (CD74) in human breast cancer. Oncol Rep 32: 523-529, 2014.

31. Tan L, Ye X, Zhou Y, Yu M, Fu Z, Chen R, Zhuang B, Zeng B, Ye H, Gao W, et al: Macrophage migration inhibitory factor is overexpressed in pancreatic cancer tissues and impairs insulin secretion function of $\beta$-cell. J Transl Med 12: 92, 2014
32. Wang D, Luo L, Chen W, Chen LZ, Zeng WT, Li W and Huang XH: Significance of the vascular endothelial growth factor and the macrophage migration inhibitory factor in the progression of hepatocellular carcinoma. Oncol Rep 31: 1199-1204, 2014.

33. Tas F, Karabulut S, Serilmez M, Ciftci R and Duranyildiz D: Serum levels of macrophage migration-inhibitory factor (MIF) have diagnostic, predictive and prognostic roles in epithelial ovarian cancer patients. Tumour Biol 35: 3327-3331, 2014.

34. Pei XJ, Wu TT, Li B, Tian XY, Li Z and Yang QX: Increased expression of macrophage migration inhibitory factor and DJ-1 contribute to cell invasion and metastasis of nasopharyngeal carcinoma. Int J Med Sci 11: 106-115, 2013.

35. Morris KT, Nofchissey RA, Pinchuk IV and Beswick EJ: Chronic macrophage migration inhibitory factor exposure induces mesenchymal epithelial transition and promotes gastric and colon cancers. PLoS One 9: e98656, 2014.

36. Ren Y, Chan HM, Fan J, Xie Y, Chen YX, Li W, Jiang GP, Liu Q, Meinhardt A and Tam PK: Inhibition of tumor growth and metastasis in vitro and in vivo by targeting macrophage migration inhibitory factor in human neuroblastoma. Oncogene 25: 3501-3508, 2006.

37. Ren Y, Chan HM, Li Z, Lin C, Nicholls J, Chen CF, Lee PY, Lui V, Bacher $\mathrm{M}$ and Tam PK: Upregulation of macrophage migration inhibitory factor contributes to induced N-Myc expression by the activation of ERK signaling pathway and increased expression of interleukin-8 and VEGF in neuroblastoma. Oncogene 23: 4146-4154, 2004 